\title{
An Agent-Based Approach to Virtual Power Plants of Wind Power Generators and Electric Vehicles
}

\author{
Matteo Vasirani ${ }^{\circ}$, Ramachandra Kota ${ }^{+}$, Renato L.G. Cavalcante ${ }^{\star}$, Sascha Ossowski*, Nicholas R. Jennings ${ }^{+}$ \\ ${ }^{\circ}$ Distributed Information Systems Laboratory, EPFL, Lausanne, Switzerland \\ * Centre for Intelligent Information Technology, University Rey Juan Carlos, Madrid, Spain \\ * Fraunhofer Institute for Telecommunications, Heinrich Hertz Institute, Berlin, Germany \\ + Electronics and Computer Science, University of Southampton, Southampton, UK \\ matteo.vasirani@epfl.ch, rck05r@ecs.soton.ac.uk, renato.cavalcante@hhi.fraunhofer.de, \\ sascha.ossowski@urjc.es, nrj@ecs.soton.ac.uk
}

\begin{abstract}
Wind power is gaining in significance as an important renewable source of clean energy. However, due to their inherent uncertainty, wind generators are often unable to participate in the forward electricity markets like the more predictable and controllable conventional generators. Given this, virtual power plants (VPPs) are being advocated as a solution for increasing the reliability of such intermittent renewable sources. In this paper, we take this idea further by considering VPPs as coalitions of wind generators and electric vehicles, where wind generators seek to use electric vehicles (EVs) as a storage medium to overcome the vagaries of generation. Using electric vehicles in this manner has the advantage that, since the number of EVs is increasing rapidly, no initial investment in dedicated storage is needed. In more detail, we first formally model the VPP and then, through an operational model based on linear programming, we show how the supply to the Grid and storage in the EV batteries can be scheduled to increase the profit of the VPP, while also paying for the storage using a novel scheme. The feasibility of our approach is examined through a realistic case-study, using real wind power generation data, corresponding electricity market prices and electric vehicles' characteristics.
\end{abstract}

Index Terms-Virtual power plants, electric vehicles, agentbased approach, linear programming

\section{INTRODUCTION}

Renewable energy has received considerable attention in recent years, and investments in this sector have been increasing steadily, especially in wind power generation. Indeed, countries such as Spain and Denmark already use wind farms to supply, respectively, $11 \%$ and $20 \%$ of their national electricity needs ${ }^{1}$. Unfortunately, despite its environmental advantages over many other sources of energy, wind power generation has many drawbacks that have limited its wider adoption. In particular, wind energy production is intermittent and prone to large forecast errors [1] — two highly undesirable features for electricity grid operators [2]. Moreover, in many regions, the peak of wind energy production is often reached at night, when demand is low. As a result, much of the energy produced by wind is sold during these periods in which electricity prices are also low [3]. Therefore, from a strictly economic point of view, wind power generation is often not competitive compared to other mature technologies of energy generation.

${ }^{1}$ See http://www.ree.es and http://www.ens.dk.
To address the above issues and to promote the development of wind farms, many countries pay wind energy providers a pre-determined feed-in tariff that is typically above market prices. However, with the expected proliferation of wind farms, these preferential policies are not desirable in the long run (indeed, there are already plans towards rolling-back the feedin tariffs in many countries, such as UK and Germany).

Against this background, in this work we study an efficient scheme that enables wind energy providers to improve their profits by participating in short-term electricity markets, such as the day-ahead market. To do so, we use storage as a means of controlling the energy supplied to the power grid (henceforth, called the 'Grid'); wind farms store the energy produced during periods of low prices and sell to the Grid during periods of high prices. This approach also enables wind generators to counter the unpredictability of wind power generation by using storage as a buffer to meet their delivery targets. In more detail, we rely on one of the key components of the Smart Grid [4], that is, the virtual power plant (VPP). A VPP can be understood as a coalition composed of multiple energy producers and, possibly, energy storage providers that come together to sell electricity as an aggregate [5]. In our context, we consider VPPs of wind farms that use electric vehicles (EVs) for storage [6]. EVs are already being considered as a suitable means of stabilising the Grid [7][8] and aiding in the integration of renewable sources under the moniker of 'Vehicle-to-Grid' (V2G) [9][10]. For us, choosing electric vehicles as the storage resource has the following advantages. First, the number of EVs is increasing rapidly, and, being connected to the Grid, EVs provide easy access to a large battery storage capacity. ${ }^{2}$ In addition, although an ICT infrastructure is needed to support the communication between the VPP members, using EVs as storage does not represent an upfront capital cost for the VPP. Another benefit of such an approach is that it can help to subsidise the cost of buying and maintaining EVs by paying for their unused storage capacity. ${ }^{3}$

The objective of this work is to study the viability of having VPPs that use EVs as a storage medium. In particular, we

\footnotetext{
${ }^{2}$ Private vehicles are typically parked $96 \%$ of their time. EVs can remain connected to the Grid during this period.

${ }^{3}$ However, it should be noted that our approach would work equally well with any other form of battery storage, and need not necessarily be EV-based.
} 
TABLE I

NOTATION

\begin{tabular}{llll}
\hline \hline Variable & Description & Variable & Description \\
\hline $\mathcal{H}$ & Wind power generation site & $\mathcal{V}$ & Set of EVs \\
$\mathbf{z}$ & Day-ahead estimated generation $(\mathrm{kWh})$ & $\mathbf{s}_{v}$ & Available storage of a single EV (kWh) \\
$\mathbf{x}$ & Energy supplied directly to the Grid $(\mathrm{kWh})$ & $D o D$ & Depth of discharge \\
$\mathbf{b}$ & Energy transferred to the batteries $(\mathrm{kWh})$ & $E^{\mathrm{max}}$ & $\mathrm{EV}$ battery capacity (kWh) \\
$\mathbf{d}$ & Energy transferred from the batteries $(\mathrm{kWh})$ & $L_{\mathrm{ET}}$ & Battery lifetime throughput energy (kWh) \\
$\mathbf{s}$ & Total available storage $(\mathrm{kWh})$ & $L(D o D)$ & Battery lifetime (\# of cycles) \\
$\mathbf{w}$ & Total energy supplied to the Grid $(\mathrm{kWh})$ & $c^{b}$ & Battery capital cost (€) \\
$\mathbf{y}$ & Needed storage capacity $(\mathrm{kWh})$ & $c^{\mathrm{EV}}$ & Cost of VPP participation $(€)$ \\
$\mathbf{g}$ & Energy transferred to the batteries as payment $(\mathrm{kWh})$ & $E^{s}(D o D)$ & Energy stored for the VPP as function of $D o D(\mathrm{kWh})$ \\
$\mathcal{P}(\mathbf{x}, \mathbf{d})$ & Profit function of the VPP $(€)$ & $E^{f}(D o D)$ & Energy received as payment as a function of $D o D(\mathrm{kWh})$ \\
$\sigma$ & Percentage of y that is transferred as payment & $\mathcal{P}^{\mathrm{EV}}(D o D)$ & Profit function of an EV $(€)$ \\
$\eta$ & Battery conversion loss & $\mathbf{p}^{e}$ & Wholesale price of electricity $(€)$ \\
$\mathbf{u}_{t}^{\text {up }}$ & Balancing-up required at time $t(\mathrm{kWh})$ & $\mathbf{p}^{\mathrm{up}}$ & Balancing-up penalty $(€)$ \\
$\mathbf{u}_{t}^{\text {down }}$ & Balancing-down required at time $t(\mathrm{kWh})$ & $\mathbf{p}^{\text {down }}$ & Balancing-down penalty $(€)$ \\
$\mathcal{B}_{t}$ & Total energy stored in the EVs at time $t(\mathrm{kWh})$ & $\epsilon_{t}$ & Wind speed forecasting error $t$ hour ahead \\
\hline \hline
\end{tabular}

define an approach for the modelling and implementation of VPPs of wind generators and EVs to participate in the electricity day-ahead market. Since the participants in the VPP are autonomous entities with private preferences that act in their own interest and interact with one another, we claim that an agent-based perspective is highly appropriate to model the functioning of a distributed system such as a VPP [11].

We also introduce a novel payment scheme to compensate $\mathrm{EVs}$ for the use of their batteries (which shortens the battery life), and to make the participation in the VPP profitable enough for EVs, guaranteeing that the VPP will actually be formed. Most importantly, we then conduct an economic feasibility analysis of our whole approach through a case study based on real data of wind generation and historical data of market prices to simulate the functioning of a VPP. The experimental evaluation shows that the formation of VPPs is economically feasible and guarantees positive gains to both wind farms and EVs.

In summary, the contributions of our work are as follows: (i) We suggest an agent-based approach for VPPs of wind generators and EVs. (ii) We extend the state of the art in the Smart Grid domain by showing how a VPP can optimise its energy supply schedule to the Grid and maximise profit using storage to cope with the uncertainty associated with the prediction of wind power generation. (iii) We present a novel payment scheme for compensating the EV agents for their participation in the VPP. (iv) We perform an analysis through a realistic case study that shows how our model permits the formation of VPPs that are profitable for both wind farms and EVs.

The article is structured as follows. In Section II, we briefly review relevant literature. Section III introduces our concept of virtual power plant, and the modelling of its members. Next, in Section IV, we define the formal model of a virtual power plant, and show how the VPP can optimise its schedule of supply to the Grid. Section V contains the details of our empirical evaluation through a case study.

\section{RELATED WORK}

The growing pressure to increase the contribution of renewable sources to electricity generation has prompted the inclusion of distributed energy resources (e.g., fuel cells, PV systems, small biopower) into many electricity systems. Given this, VPPs have recently been proposed [5] as a means of providing an appropriate interface for a set of distributed generation and storage installations to be able to deal efficiently with the Grid operators and other market actors [12]. Although the benefits of VPPs have been extensively assessed, the usual approach assumes that the components of the VPP belong to the same stakeholder that has full control over them [13]. Therefore, there is no need to take into account the preferences and incentives of the VPP components. However, a different approach is needed when each component of the VPP belongs to a different stakeholder. For this reason, agent-based techniques are starting to be considered as a suitable means to realise the VPP concept. One example is [14], wherein they propose a mechanism to promote formation of 'cooperative' VPPs of such renewable sources. However, although their scheme is intended as an improvement on the feed-in tariffs, it does not integrate the players into the actual energy markets.

Similarly, an agent-based technique for control of VPPs has been presented in [15]. They propose a three-layer architecture to control a VPP, where low layer agents are responsible for locally managing VPP components, while high layer agents look after the VPP-wide aspects like market participation and trading strategy. However, very little detail is provided about the mechanisms employed by the agents of the various layers to run the VPP.

More relevant to our context is the idea of coupling generation with storage explored by [16]. They analyse the economic performance of wind power generation by coupling a wind farm with energy storage facilities, thus constituting a virtual power plant. A method based on dynamic programming is proposed for scheduling and operating such a plant in an electricity market setting. However they do not study the more challenging scenarios whereby the storage facilities are owned by other stakeholders such as the privately owned electric vehicles in our case. Having such multiple stakeholders brings in aspects of revenue pay-offs, uncertainty and dynamism in the VPPs which need to be addressed. In their work the wind farm stakeholder owns a set of storage facilities that can be used whenever needed. Although the authors do not specify 
how much energy storage is available to the wind farm, it is evident that the investment required for this kind of storage facility is considerable, especially to be sustained by a single stakeholder. Thus, their approach in not likely to be widely adopted by wind generators.

Nevertheless, the study of agent-based techniques for EV management and $\mathrm{V} 2 \mathrm{G}$ activities has been picking up pace in recent times [17]. An agent-based approach for forming coalitions of EVs that provide the Grid with regulatory services is presented in [18]. Particularly, they attempt to address some of the challenges underlying the integration of the EVs into the Grid through coalition formation and aggregation techniques. Thus, their work is encouraging with regards to the prospect of using EVs connected to the Grid to provide storage services, while also championing the application of agentbased techniques in this domain. However, they focus purely on the regulatory market, which is significantly different from the spot market (which is our focus), and hence they also lack the perspective from the generators' side.

\section{VPP PARTICIPANTS}

As explained earlier, a VPP is composed of some wind energy producers and electric vehicles that act in the electricity market as a single entity. Now, there are several electricity markets, operating at different time scales, where conventional market players can place their bids. Future markets trade power for months-ahead or even years-ahead delivery, mainly to hedge against future price fluctuations. In day-ahead spot markets, power is traded on day $k-1$ in order to deliver it on day $k$. When the day-ahead market closes, the production schedule for the next day is determined by the grid operator. The supplier therefore establishes a contractual relationship with the grid operator that requires them to deliver a certain amount of energy for the next day and to pay for any deviation (positive or negative) to the committed schedule. During the day of delivery there is an intra-day market to reschedule generation and consumption if necessary. The balancing market takes place after the intra-day market. If a supplier is not able to deliver the contracted power, the balancing market ensures energy balance between generation and consumption. In this case, the supplier that was responsible for the deviation from the contracted schedule must pay imbalance penalties.

Since wind power prediction is sufficiently accurate only for short time horizons, in our context, we consider the scenario where a VPP participates in the day-ahead market. On day $k-1$, a VPP submits a supply bid where it commits itself to deliver a specific amount of electricity at every time-slot of day $k$. Therefore, on day $k-1$ the wind energy providers of the VPP estimate their generation based on weather predictions. Given the set of EVs that are willing to join the VPP for day $k$, wind energy providers calculate the optimal amount of storage needed to place the most profitable bids in the dayahead market. On day $k$ (i.e., the actual day of delivery), based on the realised production, the VPP continuously reoptimises the supply to maximise profit while taking into account the agreed contract. Based on this functioning model, three major research questions will be addressed in Section V: (i) What is the potential profit obtained by using the available storage? (ii) What is the total amount of storage needed to maximise such a profit? (iii) What is the potential profit made by the EVs? Next, we present the model of the wind generator and electric vehicle agents before moving onto the formal model of the VPP.

\section{A. Wind Power Generation Site}

Let $\mathcal{H}$ denote a wind power generation site (or wind farm), owned by a single stakeholder and managed by a software agent. A single wind turbine $j$ of a wind power generation site $\mathcal{H}$ is characterised by a nominal power curve that for a given wind speed (in $\mathrm{m} / \mathrm{s}$ ) returns the generated power. Let $P_{j}(t)$ be the power generated by turbine $j$ over time for a given day. The day is divided into $N$ time slots, and the expected electricity generated during the $n$th time slot is given by $Q_{j}(n)=\int_{t=n \tau}^{t=(n+1) \tau} P_{j}(t) d t$, where $\tau=24 / N$. The sum over all the wind turbines at generation site $\mathcal{H}$ gives us the generation vector, defined as $\mathbf{z}=\left[\begin{array}{lll}z(0) & z(1) \ldots z(N-1)\end{array}\right]^{T}$, where $z(n)=\sum_{j=1}^{|\mathcal{H}|} Q_{j}(n)$. This expected generation vector $\mathbf{z}$ is used for deciding the day-ahead bid (c.f. (1)). Hereafter, for notational convenience, the $n$th element of any vector $\mathbf{x}$ is denoted by $x(n)$.

\section{B. Electric Vehicles}

Let $\mathcal{V}=\left\{v_{1}, v_{2}, \ldots, v_{k}\right\}$ be a set of electric vehicles. An EV is managed by a software agent that acts on behalf of the human owner, and it is characterised by a storage profile, which defines the amount of energy that can be stored in its battery in each time slot and made available to the VPP. For a generic EV $v \in \mathcal{V}$, let $\mathbf{s}_{v}$ be the storage profile vector for the $N$ time slots that compose a day, $\mathbf{s}_{v}=\left[s_{v}(0) s_{v}(1) \ldots s_{v}(N-1)\right]^{T}$, where $s_{v}(n)$ is the quantity of energy that $v$, if included in the VPP, can store at time slot $n$. In our context, it is obvious that the storage profile vector should be calculated based on the owner's preferences (i.e., when the EV will be available to store energy, what amount of storage is offered by the EV to the VPP, etc.). In more detail, let $E^{\max }$ be the maximum energy that can be stored in the battery of the EV. Each vehicle is characterised by a preferred depthof-discharge regime $(D o D \in[0,1])$, which indicates that the vehicle is willing to provide at most $D o D \cdot E^{\max }$ units of storage. In fact, the depth-of-discharge not only affects the battery life cycle, thereby the cost of participation of the EV in the VPP, but also it affects the driving range, since the battery capacity available for driving is reduced to $(1-D o D) E^{\max } \mathrm{kWh}$. The storage profile vector is therefore defined as $\mathbf{s}_{v}=\left[D o D \cdot E^{\max } D_{o D} \cdot E^{\max } \ldots D o D \cdot E^{\max }\right]^{T}$.

\section{VPP MODEL}

In general, a VPP may contain several wind power generation sites. However, in this work, for the sake of simplicity, we assume that a VPP contains a unique wind farm, which here we assume is the VPP leader. This does not cause any loss of generality because multiple generation sites can together be represented by a single agent for the purposes of calculating 
the overall production and required storage at the VPP (which is the aim of this section).

Formally, let the estimated electricity generated by the VPP on the next-day at the $n$th time slot be $z(n) \in \mathbb{R}$. This estimated quantity $z(n)$ can be supplied directly to the Grid, stored in the batteries of the electric vehicles, or both. Furthermore, for the same time slot, the VPP leader may want to transfer to the Grid an additional quantity of electricity that was stored in the batteries of the vehicles in previous time steps. These decisions depend on the market prices and also on the cost of using storage. In the following, we will describe how EVs are paid for the storage they provide to the VPP, the optimisation model used by the VPP to define the supply bid to submit on day $k-1$ to the day-ahead market and the reoptimisation procedure executed on day $k$ to limit the imbalances caused by wind power prediction errors.

\section{A. Storage Payment Scheme}

In our approach, payment for storage is provided to the EVs in the form of charging entitlements rather than money, to take advantage of the price differential between wholesale and retail market price of electricity. This work does not consider transmission costs because, in any case, they are paid by each EV owner as part of the energy supply installation. The cost to the VPP for supplying the electricity is very low as compared to the value placed on it by the EVs. For example, typically in Spain, wholesale market prices are at $0.04 € / \mathrm{kWh}$ whereas the retail prices are around $0.14 € / \mathrm{kWh}$. Therefore, a donation of $1 \mathrm{kWh}$ to an EV will result in a loss of revenue of about $0.04 €$ to the VPP as it could have otherwise sold this energy in the market. At the same time, the perceived gain to the EV is $0.14 €$ as it would otherwise have paid that much to purchase it through a retail tariff. This way of paying for storage will help in overcoming the main perceived disadvantage of using EV storage for electricity spot markets - low revenues from the market and high cost of battery life [19]. Thus, our storage payment scheme is in the form of energy given away to the EVs by the generators. The amount of energy given away is measured as a proportion of the amount of storage used, which thereby acts as the representative of the storage cost.

Given the above, the agent leading the VPP computes the optimal schedule that maximises its profit based on predictions of energy production and storage capacity for the next day, and uses this schedule to place bids in the day-ahead market (Section IV-B). Then, on the actual day of delivery, the leader continuously reoptimises the schedule for the remainder of the day to take into account the contracted energy supply and the latest predictions of the energy production and available storage (Section IV-C).

\section{B. Day-Ahead Optimisation}

To place the bid, the leader has to compute the following five parameters that determine the supply schedule: (i) the amount supplied directly into the Grid, (ii) the amount of energy transferred to the batteries, (iii) the energy transferred from the batteries to the Grid, (iv) the amount of battery storage capacity needed (used to determine the numbers of
EVs needed in the VPP) and (v) the amount of energy transferred to the EVs as payment. These five parameters are denoted, respectively, by the vectors $\mathbf{x} \in \mathbb{R}^{N}, \mathbf{b} \in \mathbb{R}^{N}$, $\mathbf{d} \in \mathbb{R}^{N}, \mathbf{y} \in \mathbb{R}^{N}$ and $\mathbf{g} \in \mathbb{R}^{N}$. Let the maximum total storage (upper bound for $\mathbf{y}$ ) available to the VPP be given by $\mathbf{s}=\left[\begin{array}{llll}s(0) & s(1) & \ldots & s(N-1)\end{array}\right]^{T}$ and $s(n)=\sum_{v \in \mathcal{V}} s_{v}(n)$, where $\mathcal{V}$ denotes the set of EVs.

Let us denote that the electricity supplied to the Grid (either directly or drained from the batteries) is paid for at price $p^{\mathrm{e}}(n)$. Also, let the ratio between the amount of energy given to the EVs as payment and the amount of storage used be denoted by $\sigma \in[0,1]\left(\sigma \leq \frac{g(n)}{y(n)}\right)$. Finally, let $\eta \in(0,1)$ be the battery's overall conversion loss, which takes into account the percentage of electricity that is lost when electricity flows from the Grid to the battery and vice-versa. Therefore, it is necessary to store $1+\eta$ units of energy to have 1 unit actually delivered from the battery.

The objective of the VPP is solving the following optimisation problem ${ }^{4}$ :

$$
\begin{aligned}
& \underset{\mathbf{x}, \mathbf{b}, \mathbf{d}, \mathbf{y}, \mathbf{g}}{\operatorname{maximise}} \mathcal{P}(\mathbf{x}, \mathbf{d})=\sum_{n=0}^{N-1} p^{\mathrm{e}}(n)[x(n)+d(n)] \\
& \text { subject to } \\
& \forall n \in\{0, \ldots, N-1\} \\
& x(n)+(1+\eta) b(n)+g(n)=z(n), \\
& \Delta(n)+b(n) \leq y(n) \text {, } \\
& \Delta(n)-d(n) \geq 0, \\
& g(n) \geq \sigma y(n), \\
& x(n) \geq 0, b(n) \geq 0, d(n) \geq 0, y(n) \geq 0, \\
& 0 \leq y(n)+g(n) \leq s(n) \text {. }
\end{aligned}
$$

where $\mathcal{P}(\mathbf{x}, \mathbf{d})$ represents the revenues raised by the VPP from the electricity sold in the market, based on the estimated generation $\mathbf{z}$, and

$$
\Delta(n)= \begin{cases}0, & \text { if } n=0 \\ \sum_{i=0}^{n-1}(b(i)-d(i)) & \text { otherwise }\end{cases}
$$

is the net energy stored in the EVs' batteries at the beginning of time slot $n$. (We assume in (2) that the initial available energy is zero, but this can be easily generalised to the case where some energy is already present.)

The first constraint in (1) guarantees that the electricity $z(n)$ is split into a quantity that is supplied to the Grid, $x(n)$, another quantity that is stored in the batteries, $b(n)$, and the quantity that is given away to the EVs as payment for their participation, $g(n)$. The second constraint guarantees that the electricity that is stored in the batteries fits the available storage. The third constraint guarantees that the electricity that is drained from the batteries does not exceed the energy that is actually stored in the batteries. The fourth constraint

\footnotetext{
${ }^{4}$ Note that (1) is a standard linear programming (LP) problem that can be solved with any off-the-shelf LP solver. In particular, we used an implementation of the simplex method offered by the Apache Commons Math library. The typical execution to solve an instance of the problem takes no more than 2 seconds on a $2.66 \mathrm{GHZ}$ Intel Core 2 Duo with 4GB of memory.
} 
guarantees that the payment received by the EVs in terms of energy $g(n)$ is at least as much as the required proportion (given by $\sigma$ ) of storage used $y(n)$. By solving this optimisation problem, ${ }^{5}$ the day-ahead bid $\mathbf{w}$ is given by $\mathbf{w}=\mathbf{x}+\mathbf{d}$.

\section{Receding Horizon Optimisation}

During the day of delivery, the closer we get to a time slot, the better the estimate of the energy production. At any given time slot on the day of delivery, the schedule computed in Section IV-B may need to be adjusted to cope with dynamic changes (e.g., the VPP may be unable to supply the contracted energy). Therefore, the VPP leader should continuously adapt to this dynamic and uncertain setting in order to maximise profit.

The approach taken in this work is based on receding horizon optimisation [20]. Although this approach is not optimal, it is one of the most widely used techniques in practice when the problem at hand is not solvable with methods that find the optimal policy [21], such as dynamic programming (DP). In fact, straightforward DP is not only computationally expensive when the state and input space are continuous and multidimensional, but it is also impossible to use when the disturbances are not conditionally independent of the past disturbances [21]. In receding horizon optimisation, a deterministic optimal control problem is solved at each time step, using estimates available at the current time step for the unknown future disturbances. The solution to this optimisation problem yields the optimal sequence of actions for the finite horizon, if the future disturbances were equal to the estimates. Then only the first action of the sequence is applied. At the next time step, the computation is repeated using the exact value of the current state, which is now known, and an updated set of predictions for the future disturbances [20].

More formally, let $\mathbf{z}_{t}^{\prime} \in \mathbb{R}^{N}$ be the new energy forecast vector known at time slot $t$ for the remainder of the $N-t$ time slots of the day; i.e., $z_{t}^{\prime}(t), \ldots, z_{t}^{\prime}(N-1)$ are the forecasts, at time $t$, for time slots $t, \ldots, N-1$, respectively (other components of the vector $\mathbf{z}_{t}^{\prime}$ can be ignored). Similarly, let $\mathrm{s}_{t}^{\prime} \in \mathbb{R}^{N}$ represent the new maximum total storage capacity available to the VPP at time $t$ for the remainder of the day. Let $\mathbf{p}^{\text {up }}$ be the cost vector of the penalty that an electricity supplier must pay if it is "short", i.e., if it delivers less energy than that contracted in the day-ahead market. However, if the agent is "long", i.e., it provides more energy than contracted, the price of the energy surplus is not higher than that in the day-ahead contract. ${ }^{6}$ The difference in these two prices (the contracted price minus the actual surplus price) is denoted by $\mathbf{p}^{\text {down }}$. More precisely, $p^{\text {down }}(n)=p^{\mathrm{e}}(n)-p^{\text {surplus }}(n)$, where $p^{\text {surplus }}(n)$ is the price of the extra energy at the $n$th time slot. $^{7}$

\footnotetext{
${ }^{5}$ In cases where we would like to know the ideal amount of storage needed to attain the highest possible profit, we can replace the constraint $0 \leq y(n) \leq$ $s(n)$ in (1) by the constraint $0 \leq y(n)$.

${ }^{6}$ This setting prevents suppliers that participated in the day-ahead market from reserving a certain amount of power with the aim of delivering it in the balancing market at a higher price. In fact, the functionality of covering short or long positions is carried out by the reserve power suppliers, which have a different contractual relation with the grid operator.

${ }^{7}$ We assume that we cannot learn any additional information about prices during the day of delivery, so for our purposes the prices remain unchanged.
}

From this, it follows that $\mathrm{p}^{\text {down }} \geq \mathbf{0}$, where " $\geq$ " stands for the element-wise inequality.

Now, at every time slot $t$, given the contracted supply $\mathbf{w}$ (recall $\mathbf{w}=\mathbf{x}+\mathbf{d}$ ), the leader has to compute a new schedule $\mathbf{x}_{t}^{\prime}, \mathbf{b}_{t}^{\prime}, \mathbf{d}_{t}^{\prime}, \mathbf{g}_{t}^{\prime} \in \mathbb{R}^{N}$ and required storage $\mathbf{y}_{t}^{\prime} \in \mathbb{R}^{N}$, which are the updated versions of $\mathbf{x}, \mathbf{b}, \mathbf{d}, \mathbf{g}$ and $\mathbf{y}$, respectively, for the remainder of the day (the remaining $N-t$ slots). Therefore, the new supply vector $\mathbb{R}^{N} \ni \mathbf{w}_{t}^{\prime}=\mathbf{x}_{t}^{\prime}+\mathbf{d}_{t}^{\prime}$ is computed at every time $t \in\{0, \ldots, N-1\}$. The imbalance vector for being "short" is, therefore, $\mathbf{u}_{t}^{\text {up }}=[\max (0, w(t)-$ $\left.\left.w_{t}^{\prime}(t)\right) \ldots \max \left(0, w(N-1)-w_{t}^{\prime}(N-1)\right)\right]^{T}$. Similarly, the imbalance vector for being "long" is defined as $\mathbf{u}_{t}^{\text {down }}=$ $\left[\max \left(0, w_{t}^{\prime}(t)-w(t)\right) \ldots \max \left(0, w_{t}^{\prime}(N-1)-w(N-1)\right)\right]^{T}$. With the above, the profit function at time $t$ considering imbalance penalties is given by

$$
\begin{aligned}
\mathcal{P}_{t}^{\prime}\left(\mathbf{x}_{t}^{\prime}, \mathbf{d}_{t}^{\prime}\right)=\sum_{n=0}^{N-1} p^{\mathrm{e}}(n)\left[x_{t}^{\prime}(n)+d_{t}^{\prime}(n)\right] \\
\quad-\sum_{n=0}^{N-1} p^{\mathrm{up}}(n) u_{t}^{\mathrm{up}}(n)-\sum_{n=0}^{N-1} p^{\text {down }}(n) u_{t}^{\text {down }}(n) .
\end{aligned}
$$

Given that the above function, although concave, ${ }^{8}$ is not differentiable everywhere due to he max function, we recast the optimisation problem having (3) as the cost function as

$$
\begin{aligned}
\underset{\mathbf{x}_{t}^{\prime}, \mathbf{b}_{t}^{\prime}, \mathbf{d}_{t}^{\prime}, \mathbf{y}_{t}^{\prime}, \mathbf{g}_{t}^{\prime}, \alpha, \beta}{\operatorname{maximise}} & \sum_{n=t}^{N-1} p^{\mathrm{e}}(n)\left[x_{t}^{\prime}(n)+d_{t}^{\prime}(n)\right] \\
& -\sum_{n=t}^{N-1} p^{\mathrm{up}}(n) \alpha(n)-\sum_{n=t}^{N-1} p^{\text {down }}(n) \beta(n) \\
\text { subject to } & \\
\forall n \in\{0, \ldots, N-1\} & x_{t}^{\prime}(n)+(1+\eta) b_{t}^{\prime}(n)+g_{t}^{\prime}(n)=z_{t}^{\prime}(n), \\
& \mathcal{B}_{t}+\Delta_{t}^{\prime}(n)+b_{t}^{\prime}(n) \leq y_{t}^{\prime}(n), \\
& \mathcal{B}_{t}+\Delta_{t}^{\prime}(n)-d_{t}^{\prime}(n) \geq 0, \\
& g_{t}^{\prime}(n) \geq \sigma y_{t}^{\prime}(n), \\
& x_{t}^{\prime}(n) \geq 0, b_{t}^{\prime}(n) \geq 0, d_{t}^{\prime}(n) \geq 0, y_{t}^{\prime}(n) \geq 0 \\
& 0 \leq g_{t}^{\prime}(n)+y_{t}^{\prime}(n) \leq s_{t}^{\prime}(n), \\
& \alpha(n) \geq 0, \beta(n) \geq 0, \\
& \alpha(n)+x_{t}^{\prime}(n)+d^{\prime}(n) \geq x(n)+d(n), \\
& \beta(n)+x(n)+d(n) \geq x^{\prime}(n)+d^{\prime}(n) .
\end{aligned}
$$

where $\mathcal{B}_{t}$ is the total energy present in the EVs at time $t$, $\alpha(n)$ and $\beta(n)$ are variables included to remove the nondifferentiable function max of (3), and

$$
\Delta_{t}^{\prime}(n)=\left\{\begin{array}{lc}
0, & \text { if } n=t \\
\sum_{i=t}^{n-1}\left(b_{t}^{\prime}(i)-d_{t}^{\prime}(i)\right) & \text { otherwise. }
\end{array}\right.
$$

On the day of delivery, (4) is solved at every time slot $t$, and the schedule used at time $t$ is $x_{t}^{\prime}(t), b_{t}^{\prime}(t), d_{t}^{\prime}(t), g_{t}^{\prime}(t)$. The values

\footnotetext{
${ }^{8}$ Note that $-u_{t}^{\text {up }}$ and $-u_{t}^{\text {down }}$ are concave functions, so (3) can be seen as a positive sum of concave functions, which implies that the resulting sum is also a concave function [22].
} 
TABLE II

LIFETIME CYCLES FOR DIFFERENT $D O D$ VALUES

\begin{tabular}{|c|c|}
\hline $\boldsymbol{D o D}$ & cycles \\
\hline \hline 20 & 50,000 \\
\hline 40 & 12,000 \\
\hline 60 & 4000 \\
\hline 80 & 2500 \\
\hline
\end{tabular}

$x_{t}^{\prime}(n), b_{t}^{\prime}(n), d_{t}^{\prime}(n), g_{t}^{\prime}(n)$ for $n>t$ are only exercise values to enable the agent to maximise the total profit of the day (planning ahead).

\section{CAse Study}

We conducted an experimental case study using real-data to evaluate the economic feasibility of our approach and answer the questions raised in Section III. Here, we describe first the details of the data used for modelling the wind farms and the electricity market. We then follow that up with a discussion on the electric vehicles and the impact on their batteries caused by their participation in the VPP, before moving onto the results.

\section{A. Wind Farm and Market Data}

The proposed approach is not dependent on any specific setting of the wind farm in terms of nominal power or wind turbine technology. Thus, we assume a generic wind farm with 10 typical Izar-Bonus 55/130 wind turbines, each of them with a rotor diameter of $55 \mathrm{~m}$ and a nominal capacity of $1.3 \mathrm{MW}$. Prior to submitting its supply bid to the market, the $W G$ agent must estimate the expected generation vector $\mathbf{z}$, which implies forecasting the wind speed for the next 1 to 24 hours ahead. To simulate the forecasting error we proceed as follows. We collected the wind speed and the 1 to 24 hour ahead forecasts for two months, ${ }^{9}$ from September to October 2011 for a location containing a wind farm in Spain. ${ }^{10}$ We then compute the $t$ hour ahead forecasting error $\epsilon_{t}$ as $w s^{\text {real }}-w s_{t}^{\text {pred }}$, where $w s^{\text {real }}$ is the real wind speed and $w s_{t}^{\text {pred }}$ is the $t$ hour ahead prediction. For any $t \in\{1, \ldots, 24\}$, we compute the mean and variance of the forecast error $\epsilon_{t}$. Finally, we define a normal distribution of the forecast error parametrised with the aforementioned mean and variance.

To denote the actual winds, we use wind speed data for the years 2009 and 2010 from the same wind farm in Spain. In order to simulate the electricity market, we use historical prices from the Spanish electricity market operator ${ }^{11}$ for the corresponding period of 2009 and 2010. As described in Section IV, electricity is supplied to the EVs at no cost in order to pay for their storage resource and the ratio of this energy to the amount of available storage is denoted by $\sigma$.

\section{B. Electric Vehicle Data}

To model the impact of VPP participation on the EV battery life, we rely on the widely used cost model proposed by Kempton and Tomic [23]. Let $L_{\mathrm{ET}}$ be the battery lifetime

\footnotetext{
${ }^{9}$ http: //www. wunderground. com

${ }^{10}$ http: / / www. sotaventogalicia.com

${ }^{11}$ http: / / www . omel.es
}

throughput energy (in $\mathrm{kWh}$ ) for a specific depth-of-discharge regime $(D o D)$. Using their model, if the battery capital cost (in $€$ ) is $c^{b}$, the cost $c^{\mathrm{EV}}$ of VPP participation for the EV is therefore defined as:

$$
c^{\mathrm{EV}}=\frac{c^{b} E^{s}(D o D)}{L_{\mathrm{ET}}}
$$

where $E^{s}(D o D)$ is the energy that the EV stores on behalf of the VPP, which is also a function of $D o D$. Battery lifetime is often expressed in cycles, measured at a specific $D o D$, leading to the following expression of $L_{\mathrm{ET}}$ :

$$
L_{\mathrm{ET}}=L(D o D) \cdot E^{s}(D o D)
$$

Thus, the cost $c^{\mathrm{EV}}$ of VPP participation for the EV becomes:

$$
c^{\mathrm{EV}}=\frac{c^{b}}{L(D o D)}
$$

To determine the lifetime in cycles, we rely on the results presented by EPRI [19] related to the lifetime of Li-Ion batteries, whose technology is expected to be the most costefficient for V2G applications compared to Lead acid or NiMH [24]. The $D o D$ s that are considered in this paper, with their respective lifetime in cycles, are summarised in Table II.

The participation of an EV in a VPP implies that it receives a certain quantity of free electricity for the storage it provides to the VPP. Let $E^{f}(D o D)$ be the energy that the EV receives from the VPP. This electricity is assumed to be valued by the $\mathrm{EV}$ at $0.14 € / \mathrm{kWh}$, which is the electricity price for end users in Spain. The EV profit function is therefore defined as:

$$
\mathcal{P}^{\mathrm{EV}}=0.14 E^{f}(D o D)-\frac{c^{b}}{L(D o D)}
$$

Each EV is responsible for deciding the $D o D$ that it is willing to provide to the VPP, since this parameter affects the profitability for the EV of joining the VPP. Low $D o D$ s result in lower degradation of the battery, but also lower revenues (less storage offered to the VPP). High $D o D$ s lead to higher revenues but with faster degradation of battery.

In the experiments we assume that EVs are provided with a $30 \mathrm{kWh}$ Li-Ion battery, whose cost ${ }^{12}$ is $6330 €$. The battery's total conversion loss parameter $\eta$ is set to 0.27 [23].

\section{Results}

We conducted sufficient simulation runs to obtain statistically significant results (all error-bars denote $95 \%$ confidence intervals). The main focus of the experimental evaluation is on assessing the profit of the wind farm that leads the VPP and comparing it with the profit that the same wind farm would have generated if it did not have any storage available. Let $\mathcal{P}(\mathbf{x}, \mathbf{d})$ be the realised profit that the VPP obtains, after providing free electricity to EVs (as payment for storage) and facing imbalance penalties in the market. Let $\mathcal{P}(\mathbf{x}, \mathbf{d} \mid \mathbf{s}=0)$ be the profit raised by its component wind farm participating in the electricity market but without

\footnotetext{
${ }^{12}$ This value is the EPRI estimate of a Li-Ion battery cost in a mass production scenario [19].
} 


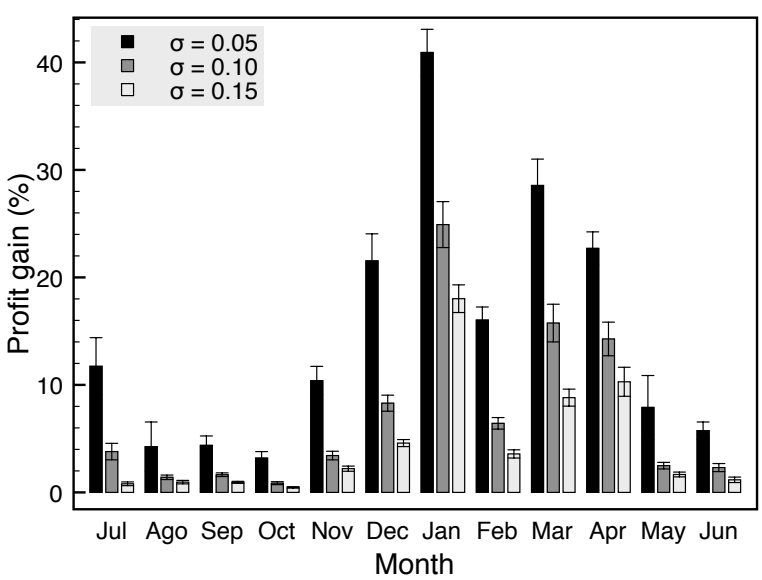

(a) Gain in profit, for different values of $\sigma$

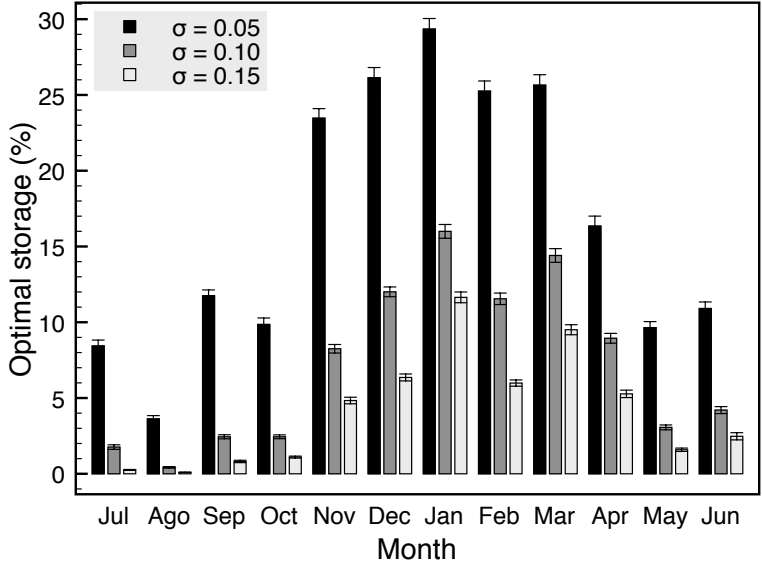

(b) Optimal storage required, for different values of $\sigma$

Fig. 1. Profit Gain and Optimal Storage needed by the VPP

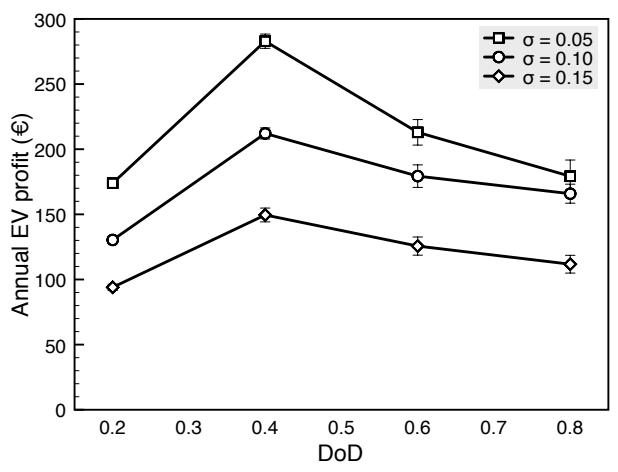

Fig. 2. Profit of an Electric Vehicle

storage, i.e., bidding according to the expected supply and facing possible imbalance penalties. The profit gain of our approach is then given by:

$$
\frac{\mathcal{P}(\mathbf{x}, \mathbf{d})-\mathcal{P}(\mathbf{x}, \mathbf{d} \mid \mathbf{s}=0)}{\mathcal{P}(\mathbf{x}, \mathbf{d} \mid \mathbf{s}=0)}
$$

In more detail, Fig. 1(a) plots the profit gain according to the month of the year, grouped by different values of $\sigma$. The profit gain tends to be higher during the winter and spring months, when the wind generation output is at its highest levels. We evaluated three values of $\sigma(0.05,0.1$ and 0.15$)$. When $\sigma$ is 0.05 , since storage is relatively cheap, the VPP uses the storage widely to maximise the profit, thus outperforming the traditional wind farm by more than $20 \%$ (as in December and March), with peaks of more than $40 \%$ of profit gain in January. The VPP is able to shift the supply towards the more profitable time slots, at noon and in the evening, drawing on the energy stored in the batteries in the previous time slots. As $\sigma$ increases, storage becomes more expensive and therefore less utilised by the VPP. As expected, the profit gain tends to shrink for $\sigma=0.10$ and $\sigma=0.15$.

Another research objective was assessing the amount of storage needed to maximise such a profit. To this end, Fig. 1(b) plots the storage required by a VPP, as a percentage of its electricity generation, to maximise its profit (note that the error

bars are too close to be visible). As expected, the amount of storage needed by the VPP decreases as it becomes more expensive due to the optimisation (in Sec. IV). In absolute terms, if we consider the winter month with the highest level of demand in terms of storage (January), the VPP must have at its disposal a storage capacity ranging from approximately $50 \mathrm{MWh}$ for $\sigma=0.05$ to $19 \mathrm{MWh}$ for $\sigma=0.15$. This observation leads us to the following question: how many electric vehicles are needed to realise this optimal storage capacity? The answer depends on the storage capacity $(D o D)$ offered by the EVs to the VPP. If we assume that each EV offers the most profitable $D o D$, which is 0.4 (see Fig. 2), a single $\mathrm{EV}$ is able to provide $0.4 \cdot 30=12 \mathrm{kWh}$, and thus, the VPP would need from 1583 to $4166 \mathrm{EVs}$ to store $19 \mathrm{MWh}$ to $50 \mathrm{MWh}$ of energy. A wind farm such as that simulated in this article, with a nominal capacity of $10 \cdot 1.3 \mathrm{MW}=13 \mathrm{MW}$, would represent $0.0006 \%$ of the Spanish installed wind power capacity, which is $21,673 \mathrm{MW} .{ }^{13}$ Given that in Spain there are $22,145,000$ passenger cars,${ }^{14}$ if we assume a $10 \%$ penetration of EVs the wind farm would need from $0.0007 \%$ to $0.0019 \%$ of the available EVs.

Finally, we also assessed the benefit to the EVs for participating in a VPP. To this end, Fig. 2 plots the EV annual profit (based on the analysis in Sec V-B) for different values of $\sigma$ and $D o D$. When $\sigma=0.05$, storage is relatively cheap and therefore the EVs are widely used. Although the price paid to the EV is lower (less electricity is provided at no cost), it is more likely that the VPP needs storage. As a result, EVs make small but frequent profits throughout the year leading to high annual profits. However, when $\sigma$ increases, the usage of storage is less profitable to the VPP. Thus, with EVs being utilised far less frequently, their annual profits are also reduced. Hence we see that, despite being counter-intuitive, a lower $\sigma$, because of the frequent use of storage, results in higher annual profits to the EVs, even overcoming the cost of the loss in battery life-cycles. Depending on the $D o D$, an $\mathrm{EV}$ is able to gain up to $282 €$ per year. It is also interesting

\footnotetext{
${ }^{13}$ http://www.aeeolica.org

${ }^{14}$ See "Energy, transport and environment indicators", Eurostat, 2010
} 
to notice how, for any given $\sigma, D o D=0.4$ is the value that maximises the EV profit. Offering even more storage is not good for an EV as costs outpace revenues.

\section{CONCLUSIONS}

The focus of this work is to enable wind power generators to fully participate in electricity markets by forming VPP with EVs that can store the energy to overcome the intermittent nature of the energy supply. We showed how the VPP can maximise its profits by optimising the schedule of supply to the Grid based on the energy production and the available storage. We also introduce a novel scheme of paying the EVs for their storage through supplying energy at no cost. Then, using real data from a wind farm, historical data of market prices and EV characteristics, we conducted a case-study to assess the economic viability of such an approach. Our analysis showed that our VPP method is more profitable to both wind farms and EVs compared to not participating in the VPP. Receding horizon optimisation showed to be simple and computationally cheap since, at each time step, a linear optimisation problem was solved using standard LP techniques. Moreover, it also delivered a control policy that guaranteed a profit for all VPP participants. These initial results are encouraging and there is significant potential for even more improvement if more advanced optimisation techniques are employed.

This work is the first assessment of the feasibility of applying the $\mathrm{V} 2 \mathrm{G}$ concept in the electricity spot markets, and therefore, opens up several avenues of future work. Specifically, such VPPs will need to have appropriate mechanisms to classify EVs based on reliability and incentivise them to adhere to their schedules. Similarly, protocols for formation and management of the VPPs need to be explored, including which EVs should be chosen for storage in any particular time period. In this respect, the notion of trust and reputation will be essential for the VPP leader to track the adherence of an $\mathrm{EV}$ to its published profile during the selection of potential VPP members.

While in our work, the case study focussed on wind farms and EVs, the basics of the approach presented here can readily be applied to other similar forms of renewable energy (e.g., photovoltaic) and storage systems (e.g., supercapacitors and flywheels). Furthermore, new actors can be modelled, such as aggregators that represent fleets of EVs and offer additional services other than storage for wind power generators, such as frequency regulation for the grid operator [25] or storage capacity for demand side management and outage management in buildings [26].

Finally, for VPPs to become a reality, a number of technological advances are required (i.e., smart meters and battery charging controllers). Many of these technological developments are already underway and this rapidly advancing field presents an attractive opportunity for agent technologies to demonstrate their utility in real-world applications [4].

\section{ACKNOWLEDGMENTS}

Work partially supported by the Spanish Ministry of Science and Innovation through the projects OVAMAH (grant
TIN2009-13839-C03-02; co-funded by Plan E) and AT (grant CSD2007-0022; CONSOLIDER-INGENIO 2010) and by the Spanish Ministry of Economy and Competitiveness through the project iHAS (grant TIN2012-36586-C03-02).

\section{REFERENCES}

[1] G. Giebel, R. Brownsword, and G. Kariniotakis, "The state-of-the-art in short-term prediction of wind power: A literature overview," in Project ANEMOS D1.1, 2003.

[2] R. Piwko, D. Osborn, R. Gramlich, G. Jordan, D. Hawkins, and K. Porter, "Wind energy delivery issues," IEEE Power \& Energy Magazine, vol. 3, no. 6, pp. 47-56, 2005.

[3] J. F. Manwell, J. G. McGowan, and A. L. Rogers, Wind Energy Explained: Theory, Design and Application. John Wiley \& Sons, 2002.

[4] S. D. Ramchurn, P. Vytelingum, A. Rogers, and N. R. Jennings, "Putting the 'smarts' into the smart grid: A grand challenge for artificial intelligence," Communications of the ACM, vol. 55, no. 4, pp. 86-97, 2012.

[5] D. Pudjianto, C. Ramsay, and G. Strbac, "Virtual power plant and system integration of distributed energy resources," Renewable Power Generation, vol. 1, no. 1, pp. 10-16, 2007.

[6] J. Lassila, J. Haakana, V. Tikka, and J. Partanen, "Methodology to analyze the economic effects of electric cars as energy storages," IEEE Transactions on Smart Grid, vol. 3, no. 1, pp. 506-516, 2012.

[7] E. Sortomme and M. A. El-Sharkawi, "Optimal scheduling of vehicle-togrid energy and ancillary services," IEEE Transactions on Smart Grid, vol. 3, no. 1, pp. 351-359, 2012.

[8] H. Sekyung, H. Soohee, and K. Sezaki, "Estimation of achievable power capacity from plug-in electric vehicles for $\mathrm{v} 2 \mathrm{~g}$ frequency regulation: Case studies for market participation," IEEE Transactions on Smart Grid, vol. 2, no. 4, pp. 632-641, 2011.

[9] W. Kempton and J. Tomić, "Vehicle-to-grid power implementation: From stabilizing the grid to supporting large-scale renewable energy," Journal of Power Sources, vol. 144, no. 1, pp. 280-294, 2005.

[10] D. P. Tuttle and R. Baldick, "The evolution of plug-in electric vehiclegrid interactions," IEEE Transactions on Smart Grid, vol. 3, no. 1, pp. 500-505, 2012.

[11] A. Rogers, S. D. Ramchurn, and N. R. Jennings, "Delivering the smart grid: Challenges for autonomous agents and multi-agent systems research," in The Twenty-Sixth AAAI Conference on Artificial Intelligence (AAAI-2012), 2012, pp. 2166-2172.

[12] E. Mashhour and S. M. Moghaddas-Tafreshi, "Bidding strategy of virtual power plant for participating in energy and spinning reserve markets - part i: Problem formulation," IEEE Transactions on Power Systems, vol. 26, no. 2, pp. 949-956, 2011.

[13] — - "Bidding strategy of virtual power plant for participating in energy and spinning reserve markets - part ii: Numerical analysis," IEEE Transactions on Power Systems, vol. 26, no. 2, pp. 957-964, 2011.

[14] G. Chalkiadakis, V. Robu, R. Kota, A. Rogers, and N. R. Jennings, "Cooperatives of distributed energy resources for efficient virtual power plants," in The Tenth International Conference on Autonomous Agents and Multiagent Systems (AAMAS-2011), 2011, pp. 787-794.

[15] A. L. Dimeas and N. D. Hatziargyriou, "Agent based control of virtual power plants," in International Conference on Intelligent Systems Applications to Power Systems (ISAP-2007), 2007, pp. 1-6.

[16] L. M. Costa, F. Bourry, J. Juban, and G. Kariniotakis, "Management of energy storage coordinated with wind power under electricity market conditions," in The Tenth International Conference on Probabilistic Methods Applied to Power Systems (PMAPS-2008), 2008, pp. 1-8.

[17] E. Gerding, V. Robu, S. Stein, D. Parkes, A. Rogers, and N. R. Jennings, "Online mechanism design for electric vehicle charging," in The Tenth International Conference on Autonomous Agents and Multiagent Systems (AAMAS-2011), 2011, pp. 811-818.

[18] S. Kamboj, W. Kempton, and K. S. Decker, "Deploying power gridintegrated electric vehicles as a multi-agent system," in The Tenth International Conference on Autonomous Agents and Multiagent Systems (AAMAS-2011), 2011, pp. 13-20.

[19] M. Duvall and M. Alexander, "Batteries for electric drive vehicles status 2005," Electric Power Research Institute, Tech. Rep., 2005.

[20] W. H. Kwon, S. Han, and S. H. Han, Receding Horizon Control: Model Predictive Control for State Models, ser. Advanced Textbooks in Control and Signal Processing. Springer, 2005.

[21] J. Skaf, S. Boyd, and A. Zeevi, "Shrinking-horizon dynamic programming," International Journal of Robust Nonlinear Control, vol. 20, pp. 1993-2002, 2010. 
[22] S. Boyd and L. Vandenberghe, Convex Optimization. Cambridge University Press, 2003.

[23] W. Kempton and J. Tomić, "Vehicle-to-grid power fundamentals: Calculating capacity and net revenue," Journal of Power Sources, vol. 144, no. 1, pp. 268-279, 2005.

[24] C. Zhou, K. Qian, M. Allan, and W. Zhou, "Modeling of the cost of ev battery wear due to v2g application in power systems," IEEE Transactions on Energy Conversion, vol. 26, no. 4, pp. 1041-1050, 2011.

[25] C. Quinn, D. Zimmerle, and T. H. Bradley, "The effect of communication architecture on the availability, reliability, and economics of plug-in hybrid electric vehicle-to-grid ancillary services," Journal of Power Sources, vol. 195, pp. 1500-1509, 2010.

[26] C. Pang, P. Dutta, and M. Kezunovic, "Bevs/phevs as dispersed energy storage for v2b uses in the smart grid," IEEE Transactions on Smart Grid, vol. 3, no. 1, pp. 473-482, 2012. 